\title{
The role of vacuum-assisted closure (VAC) therapy in the management of FOURNIER'S gangrene: a retrospective multi-institutional cohort study
}

\author{
Valerio lacovelli ${ }^{1,2} \cdot$ Chiara Cipriani $^{1} \cdot$ Marco Sandri $^{3} \cdot$ Roberta Filippone $^{2} \cdot$ Antonella Ferracci $^{4} \cdot$ Salvatore Micali $^{5}$. \\ Bernardo Rocco ${ }^{5}$. Stefano Puliatti ${ }^{5} \cdot$ Paolo Ferrarese $^{6} \cdot$ Giuseppe Benedetto $^{6} \cdot$ Andrea Minervini $^{7} \cdot$ Andrea Cocci $^{7}$. \\ Antonio Luigi Pastore ${ }^{8} \cdot$ Yazan AI Salhi $^{8}$. Alessandro Antonelli ${ }^{9} \cdot$ Tonino Morena $^{9} \cdot$ Alessandro Volpe $^{10}$. \\ Filippo Poletti ${ }^{10}$. Antonio Celia ${ }^{11}$. Guglielmo Zeccolini ${ }^{11}$. Costantino Leonardo ${ }^{12}$. Flavia Proietti ${ }^{12}$. \\ Enrico Finazzi Agrò ${ }^{2}$ P Pierluigi Bove ${ }^{1}$
}

Received: 21 January 2020 / Accepted: 14 March 2020 / Published online: 31 March 2020

(c) Springer-Verlag GmbH Germany, part of Springer Nature 2020

\begin{abstract}
Purpose To explore the role of vacuum assisted closure (VAC) therapy versus conventional dressings in the Fournier's gangrene wound therapy.

Patients and Methods This is a retrospective multi-institutional cohort study. Data of 92 patients from nine centers between 2007 and 2018 were retrospectively analyzed. After surgery, patient having a local or a disseminated FG were managed with VAC therapy or with conventional dressings. The 10-weeks wound closure cumulative rate and OS were analyzed.

Results Of the 92 patients, 62 (67.4\%) showed local and 30 (32.6\%) a disseminated FG. After surgery, 19 patients (20.7\%) with local and 14 (15.2\%) with disseminated FG underwent to VAC therapy; 43 (46.7\%) with local and 16 (17.4\%) with disseminated FG were treated using conventional dressings. The multivariable logistic regression analysis demonstrated that the VAC in patients with disseminated FG led to a higher cumulative rate of wound closure than patients treated with no-VAC $(\mathrm{OR}=6.5$; 95\% CI 1.1-37.4, $p=0.036)$. The Kaplan-Meier survival curves for the OS showed a significant difference between no-VAC patients with local and disseminated FG (OS rate at 90 days 0.90, 95\% CI 0.71-0.97 vs 0.55 , 95\% CI 0.24-0.78, respectively; $p=0.039)$. Cox regression confirmed that no-VAC patients with disseminated FG showed the lowest OS (hazard ratio adjusted for sex and age HR $=3.4,95 \%$ CI $1.1-10.4 ; p=0.033$ ).

Conclusions In this large cohort study, VAC therapy in patients with disseminated FG may offer an advantage in terms of 10 -weeks wound closure cumulative rate and OS at 90 days after initial surgery.
\end{abstract}

Keywords Fournier's gangrene $\cdot$ Necrotizing fasciitis $\cdot$ Vacuum-assisted closure therapy $\cdot$ VAC $\cdot$ Wound therapy $\cdot$ Overall survival

Valerio Iacovelli

valerio.iacovelli85@gmail.com

1 Urology Unit, San Carlo Di Nancy Hospital, GVM Care and Research, Via Aurelia 275, 00100 Rome, Italy

2 Department of Surgical Sciences, University Tor Vergata, Roma, Italy

3 Data Methods and System Statistical Laboratory, University of Brescia, Brescia, Italy

4 Department of Surgery, Policlinico Tor Vergata Foundation, Rome, Italy

5 Department of Urology, Ospedale Policlinico E Nuovo Ospedale Civile S.Agostino Estense Modena, University of Modena and Reggio Emilia, Modena, Italy
6 Urology Unit, San Bortolo Hospital, Vicenza, Italy

7 Department of Urology, University of Florence, Careggi Hospital, Florence, Italy

8 Department of Urology, ICOT Latina, Latina, Italy

9 Urology Unit, Department of Surgery, Dentistry, Paediatrics and Gynaecology, University Hospital of Verona, Verona and Confortini, Borgo General Hospital, Trento, Italy

10 Department of Urology, University of Eastern Piedmont, Maggiore Della Carità Hospital, Novara, Italy

11 Department of Urology, San Bassiano Hospital, Bassano del Grappa, Italy

12 Department of Urology, La Sapienza University of Rome, Rome, Italy 


\section{Introduction}

Since its first description in 1883, Fournier's gangrene (FG) has been defined as an aggressive necrotizing fasciitis affecting the perineal, perianal or genital areas, characterized by an acute onset, a rapid progression, potentially fatal outcomes and a high mortality rate of $20-30 \%$ [1-5]. A polymicrobial aetiology has been recognized and intentional or accidental trauma has been proposed as a possible source of infection [6, 7]. In addition, several predisposing factors such as diabetes, chronic alcoholism, human immunodeficiency virus, chronic steroid abuse, cytotoxic drugs, lympho-proliferative disease, malnutrition and lower socio-economic status have been associated with the occurrence of FG $[8,9]$.

Following the European Association of Urology (EAU) guidelines on Urological Infections [10], the initial approach must be an aggressive, complete and early $(<24 \mathrm{~h})$ surgical debridement of the necrotic tissue, as delayed and/or inadequate surgery may result in higher mortality. Immediate empiric parenteral antibiotic treatment should be given; this can then be refined, guided by microbiological culture $[8,10]$. Usually, repeated debridement is necessary leading to complex wound closure that requires frequent dressing and may need flaps or skin grafts [10]. Different protocols have been reported for postoperative open wound care, but their efficacy has not been properly analyzed and shows a very low level of evidence in the current guidelines [10]. Lack of evidence regarding the role of vacuum-assisted closure (VAC) therapy in FG (level of evidence 4) [10] led us to explore its role in this rare niche of patients.

Therefore, the aim of our retrospective analysis was to assess with a hypothesis-generating and exploratory purpose the possible role of VAC versus conventional dressings in the wound therapy of FG in terms of cumulative rate of wound closure and overall survival (OS).

\section{Patients and methods}

Data of 92 patients admitted for FG in the emergency departments of nine Italian Hospitals between March 2007 and June 2018 were retrospectively collected. All the patients received surgical treatment soon after the FG was diagnosed by the evidence of a necrotizing fasciitis involving the perineum, perianal region and/or the external genitalia [10].

The observed clinical and pathophysiologic patients' characteristics were the following: gender, age, predisposing factors, origin of FG, blood results on admission, FG extension, causative pathogens, urinary or fecal diversion, complications, length of hospital stay (LOS), type and number of surgical procedures, use of VAC or conventional dressing, time from initial debridement to wound closure. According to Ozturk et al. on the base of the FG extension, patients were classified as having a local lesion confined to the perineum and/or pubic regions or a disseminated lesion extended out of the perineum and/or pubic regions, i.e., abdomen, lumbar region or legs [11].

All patients had a multimodal approach, which included hemodynamic stabilization, surgical debridement and empiric antimicrobial therapy at admission $(<12 \mathrm{~h})$. After debridement, conventional antiseptic dressings were used to cover the wounds (no-VAC group). Hydrogen peroxide and povidone/iodine solution were mostly used to soak the dressings. As no standardized procedure has been previously described, conventional dressing depended on the single center experience. Although different antiseptic solutions were used, all the centers continued by washing repeatedly with saline until healthy granulation tissue was formed. Wound dressings were changed daily in all cases. When available in the hospital, VAC was used on the base of surgeon clinical judgement right after the surgical debridement (VAC Group). VAC was applied at $75-125 \mathrm{mmHg}$, with 5 min of suction followed by 2 min of rest, from the application until the next dressing change. For each VAC change, wounds were serially debrided under anesthesia in the operating room until healthy and viable tissue was visible. These data were not achievable for the no-VAC group given the not standardized and large heterogeneity of dressing procedures. We considered patients who were allowed to heal by secondary or tertiary (VAC-mediated) intention. All the patients who underwent reconstructive surgery (split thickness skin graft reconstruction, rotational flap reconstruction) were excluded from the retrospective analysis.

\section{Statistical analysis}

Categorical variables were summarized by absolute and relative percentage frequencies and were compared across independent groups by the Fisher's exact test. Numerical variables were summarized by median and interquartile range (IQR) and were compared across independent groups by the Wilcoxon rank sum test.

Wound closure, defined as the complete re-epithelialization of the wound surface with no discernable exudate and without drainage or dressing, was investigated in the four treatment groups using cumulative incidence functions (CIFs). Ten weeks was chosen as a reference time for assessing wound closure arbitrarily given the unavailability of standardized benchmarks. The 10-week wound closure cumulative rate was compared between groups by Fisher's 
exact test and by multivariable logistic regression (with gender and age as potential confounders).

Overall survival (OS) was defined as the time elapsed from the date of admission to the date of death from any cause or the date of last follow-up. OS was compared between groups using Kaplan-Meier survival estimates, the log-rank test, and the proportional-hazards Cox regression model.

All the statistical tests were two-sided and a p value of less than 0.05 was considered statistically significant. All estimations were performed using Stata 15 (StataCorp 2017, College Station, TX) and R 3.6.1 (R Foundation for Statistical Computing, Vienna, Austria) software.

\section{Results}

Of the 92 patients, $62(67.4 \%)$ showed local FG and 30 $(32.6 \%)$ a disseminated condition. The patients' characteristics and demographic have been listed in Table 1 according to the extension of disease and by VAC or no-VAC treatment. Of all patients, 89 (96.7\%) were males. Several characteristics of patients with local and disseminated FG managed by VAC or no-VAC treatment were similar. Particularly, no statistically significant differences were found for the following variables (see Table 1): age, distribution of predisposing factors such as obesity (BMI $\geq 30$ ), smoking, alcoholism, kidney failure, neurogenic bladder and pelvic radiotherapy and blood results such as $\mathrm{C}$-reactive protein, neutrophil-to-lymphocyte ratio and procalcitonin. The Fournier Gangrene Severity Index (FGSI) was calculated in all the groups and did not show significant differences $(p=0.13)$. The most common origin of local FG was urogenital diseases whilst disseminated FG origin was mostly mixed urogenital and anorectal. A median number of two microorganisms was isolated both in patients with local (IQR 1-3) and with disseminated FG (IQR 2-3) ( $p=0.284)$. The frequency distribution of reported microorganisms in the two FG groups are shown in Fig. 1.

The most common microorganisms reported both in patients with local FG and with disseminated FG included: Escherichia coli, Enterococcus faecalis, Pseudomonas

Table 1 Characteristics and demographic of patients with local and disseminated FG managed by VAC or no-VAC treatment

\begin{tabular}{|c|c|c|c|c|c|}
\hline & \multicolumn{2}{|l|}{ Local FG } & \multicolumn{2}{|l|}{ Disseminated FG } & \multirow[t]{2}{*}{$p$ value* } \\
\hline & VAC group & No-VAC group & VAC group & No-VAC group & \\
\hline Age, years $(n=92)$ & $62(54-75)$ & $69(58-76)$ & $66.5(58-72)$ & $70(60.5-82)$ & 0.444 \\
\hline Gender, $\mathrm{M}(n=92)$ & $19(100)^{\circ}$ & $43(100)$ & $12(85.7)$ & $15(93.8)$ & 0.027 \\
\hline \multicolumn{6}{|l|}{ Predisposing factors } \\
\hline Obesity $(n=86)$ & $6(35.3)$ & $8(20)$ & $1(7.1)$ & $3(20)$ & 0.316 \\
\hline Diabetes mellitus type II $(n=92)$ & $12(63.2)$ & $15(34.9)$ & $9(64.3)$ & $12(75)$ & 0.017 \\
\hline Hypertension $(n=92)$ & $12(63.2)$ & $19(44.2)$ & $13(92.9)$ & $12(75)$ & 0.005 \\
\hline Immobility $(n=92)$ & $4(21.1)$ & $8(18.6)$ & $8(57.1)$ & $6(37.5)$ & 0.036 \\
\hline Smoking $(n=87)$ & $9(50)$ & $20(51.3)$ & $5(35.7)$ & $6(37.5)$ & 0.671 \\
\hline Alcoholism $(n=84)$ & $3(16.7)$ & $7(18.9)$ & $3(21.4)$ & $6(40)$ & 0.393 \\
\hline Kidney failure $(n=91)$ & $7(38.9)$ & $9(20.9)$ & $2(14.3)$ & $3(18.8)$ & 0.376 \\
\hline Neurogenic bladder $(n=91)$ & $0(0)$ & $2(4.7)$ & $0(0)$ & $1(6.3)$ & 0.866 \\
\hline Pelvic radiotherapy $(n=91)$ & $2(11.1)$ & $0(0)$ & $0(0)$ & $0(0)$ & 0.089 \\
\hline \multicolumn{6}{|l|}{ Origin of Fournier's Gangrene } \\
\hline Anorectal $(n=83)$ & $2(10.5)$ & $2(5.1)$ & $3(30)$ & $3(20)$ & 0.000 \\
\hline Urogenital $(n=83)$ & $14(73.7)$ & $28(71.8)$ & $1(10)$ & $2(13.3)$ & \\
\hline Mixed (anorectal + urogenital) $(n=83)$ & $3(15.8)$ & $9(23.1)$ & $6(60)$ & $10(66.7)$ & \\
\hline \multicolumn{6}{|l|}{ Blood results on admission } \\
\hline C-reactive protein $(\mathrm{mg} / \mathrm{L})(n=87)$ & $138.7(93.7-187.0)$ & $63.7(9.6-195.0)$ & $192.6(45.0-309.0)$ & $47.2(19.6-223.8)$ & 0.154 \\
\hline Neutrophil-to-lymphocyte ratio $(n=66)$ & $14.4(9.6-19.3)$ & $12.1(8.8-18.0)$ & $12.3(8.2-36.1)$ & $16.1(6.4-39.1)$ & 0.791 \\
\hline Procalcitonin $(\mathrm{ng} / \mathrm{mL})(n=62)$ & $0.8(0.6-9.1)$ & $2.3(0.3-23.0)$ & $22.0(6.9-31.9)$ & $10.8(0.8-22.2)$ & 0.140 \\
\hline Albumin $(\mathrm{g} / \mathrm{dL})(n=84)$ & $3.0(2.7-3.2)$ & $2.8(2.2-3.3)$ & $2.6(2.0-2.6)$ & $2.3(2.0-2.9)$ & 0.008 \\
\hline FGSI & $3(1-8)$ & $3.6(1-6)$ & $2(2-6)$ & $3.7(5-9)$ & 0.135 \\
\hline
\end{tabular}

${ }^{\circ}$ No. pts $(\%)$

$n$ no. pts for which this data was recorded, $M$ males

${ }^{*} p$ value refers to a median score comparison in case of continuos variables (Kruskal-Wallis equality-of-populations rank test) or to a proportion comparison of the four groups in case of binary variables (Fisher's exact test) 


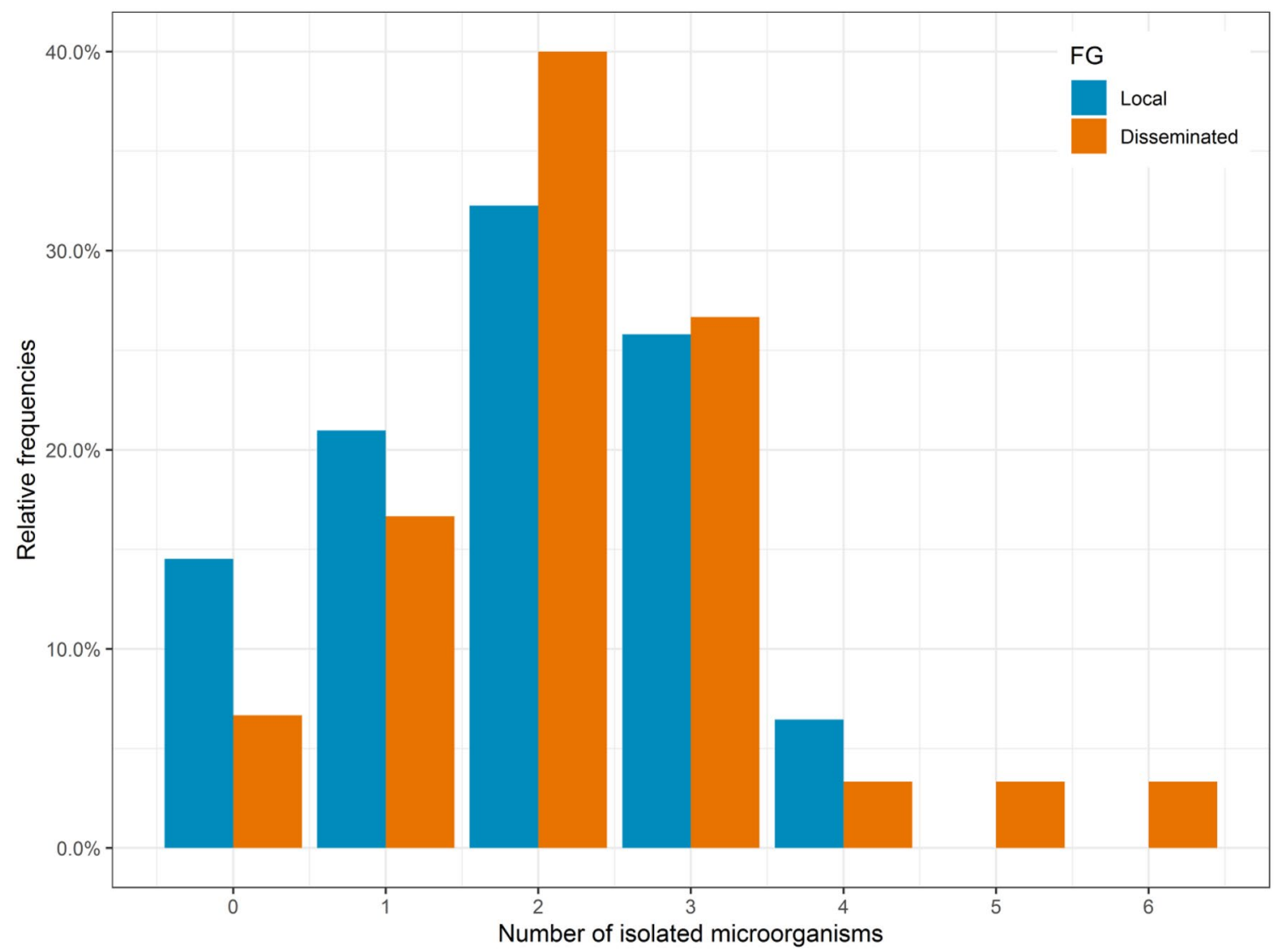

Fig. 1 Relative frequency distribution for the number of microorganisms detected in patients with local and disseminated FG

aeruginosa, Proteus mirabilis, Candida albicans, Klebsiella pneumoniae, Enterococcus faecium and Acinetobacter baumannii as listed in Table 2. All the patients underwent surgical radical debridement within the first $12 \mathrm{~h}$ after admission. The different surgical approaches used for patients with local and disseminated FG are summarized in Table 2.

Following the initial debridement, 19 patients $(20.7 \%)$ with local FG were managed with VAC therapy and 43 $(46.7 \%)$ were treated using conventional dressings. Among the disseminated FG patients, 14 (15.2\%) were managed with VAC therapy both in genital and non-genital wounds and $16(17.4 \%)$ were treated using conventional dressings. After surgery, the VAC was changed both in the local (median 6 days; IQR 1-7) and in the disseminated FG group (median 3.5 days; IQR 2-9). The median LOS was significantly longer for patients with local and disseminated FG in the VAC group (28 days, IQR 16-51, and 39 days, IQR 28-74, respectively) than in no-VAC group (18 days, IQR 12-26, and 30 days, IQR 16.5-66.5, respectively) $(p=0.006)$.

Major complications (i.e., sepsis, renal failure, acute respiratory distress syndrome, multiple organ failure) occurred both in patients with local FG (VAC group median 2.0, IQR 1.5-4.0; no-VAC group median 2.0, IQR 0.0-3.0) and in those with a disseminated condition (VAC group median 3.0, IQR 2.5-4.5; no-VAC group median 4.0, IQR 2.0-5.0) $(p=0.011)$. Overall mortality rate was $13 / 92$ patients (14.1\%). Follow-up in the entire cohort was 33 weeks, IQR 19-61.

The median time from the initial debridement to the complete wound closure was longer in patients with local FG managed with VAC therapy (45 days, IQR 30-60) than in the no-VAC group (23 days, IQR 12-36) $(p=0.011)$. In patients with disseminated FG, the median time in the noVAC group (56 days, IQR 17-90.5) was longer than in VAC group (38.5 days, IQR 23-59.5) but the difference was not statistically significant $(p=0.671)$.

Ten weeks after the initial debridement, the wound closure occurred in 14 patients $(73.7 \%)$ with local disease managed with VAC and in $33(89.2 \%)$ patients treated with noVAC approach $(p=0.247)$. In the group with disseminated disease, the wound closure was achieved in ten patients (71.4\%) managed with VAC and in four (28.6\%) treated with no-VAC approach ( $p=0.057)$ (Fig. 2).

The multivariable logistic regression analysis of 10 weeks wound closure cumulative rate demonstrated that the VAC in patients with disseminated FG led to a higher rate of wound closure than no-VAC patients (odds ratio adjusted for sex 
Table 2 Detected microorganisms and surgical approaches used in patients with local and disseminated FG

\begin{tabular}{lll}
\hline & Local FG & Disseminated $\mathrm{FG}^{\circ}$ \\
\hline $\begin{array}{l}\text { Number of patients } \\
\text { Detected microorganisms }\end{array}$ & 62 & 30 \\
Escherichia coli & $35(56.5)$ & $14(46.7)$ \\
Enterococcus faecalis & $18(29)$ & $12(40)$ \\
Pseudomonas aeruginosa & $13(21)$ & $12(40)$ \\
Proteus mirabilis & $10(16.1)$ & $2(6.7)$ \\
Candida albicans & $9(14.5)$ & $4(13.3)$ \\
Klebsiella pneumoniae & $8(12.9)$ & $4(13.3)$ \\
Enterococcus faecium & $6(9.7)$ & $6(20)$ \\
Acinetobacter baumannii & $4(6.5)$ & $3(10)$ \\
Surgical approaches & & $17(56.7)$ \\
Scrotal surgery & $55(88.7)$ & $2(6.7)$ \\
Monolateral orchiectomy & $17(27.4)$ & $2(6.7)$ \\
Bilateral orchiectomy & $5(8.1)$ & $1(3.3)$ \\
Funiculectomy & $11(17.7)$ & $14(46.7)$ \\
Perineal surgery & $16(25.8)$ & $2(6.7)$ \\
Inguinal surgery & $3(4.8)$ & $5(16.7)$ \\
Abdominal surgery & & $14(46.7)$ \\
Penile amputation & $1(1.6)$ & $3(10)$ \\
Colostomy & $6(9.7)$ & $6(20)$ \\
Ileostomy & & \\
Suprapubic cystostomy & $2(3.2)$ & \\
\hline
\end{tabular}

${ }^{\circ}$ No. pts (\%)

and age $\mathrm{OR}=6.5 ; 95 \%$ CI 1.1-37.4, $p=0.036$ ). Conversely, in patients with local FG, the 10-week wound closure cumulative rate in no-VAC patients did not show significant differences than in those managed with VAC therapy (adjusted $\mathrm{OR}=0.24 ; 95 \%$ CI $0.05-1.14, p=0.073$ ). Moreover, the comparison between the effect of VAC in patients with local and disseminated $\mathrm{FG}(\mathrm{OR}=6.5$ vs $\mathrm{OR}=0.2$, respectively $)$ was statistically significant $(p=0.009)$. Finally, although type 2 diabetes and hypertension were significantly associated with a reduction of the 10 -weeks wound closure (respectively $\mathrm{OR}=0.24 ; 95 \%$ CI $0.07-0.78, p=0.017$ and $\mathrm{OR}=0.12 ; 95 \%$ CI $0.02-0.58, p=0.09$ ), the estimated ORs did not significantly change after the addition of these pathologies to the multivariable model adjusted for sex and age.

Comparing the KM Kaplan-Meier survival curves for the OS, a significant difference between patients with local and disseminated FG was found when patients were treated with conventional dressings (OS rate at 90 days $0.90,95 \%$ CI $0.71-0.97$ vs $0.55,95 \%$ CI $0.24-0.78$, respectively; $p=0.039$ ) (Fig. 3). Furthermore, Cox regression confirmed that patients with disseminated FG managed with the noVAC approach showed the lowest OS (hazard ratio adjusted for sex and age HR $=3.4,95 \%$ CI $1.1-10.4 ; p=0.033$ ).

\section{Discussion}

FG is an obliterative endarteritis of the subcutaneous arteries resulting in gangrene of the overlying skin [11]. FG is aggressive, often life threatening and polymicrobial $[8,10]$. In our analysis, independently from the extension, all the cases were sustained by a median number of two microorganisms at the same time, being Escherichia coli and Enterococcus faecalis the most common bacteria detected. Referring to the predisposing and demographic factors, our results are in line with the currently available literature $[8,10,12]$ being the adult age or elderly age, diabetes and hypertension the most common ones.

Consensus from case series suggests that surgical debridement should be early and aggressive, as delayed and/or inadequate surgery may result in higher mortality $[8,10,12]$. Computed tomography or MRI can help define para-rectal involvement, suggesting the need for bowel diversion $[8,10]$. In our study, the surgical debridement approach varied depending on the FG extension. Intuitively, more extensive abdominal surgery and bowel diversions were more common in disseminated FG patients.

Following the initial debridement, wound management is important. Yanaral et al. reported that most cases were managed with conventional dressings that contain a wide and not standardized variety of active agents such as saline, povidone iodine, potassium permanganate, Dakin's solution, enzymatic agents for wound cleansing, or polyhexanide [13]. The same authors retrospectively compared 54 FG patients treated with VAC or conventional dressings reporting that VAC was effective offering fewer dressing changes, less pain, and greater mobility [13]. The role of VAC therapy has been furtherly investigated in several case series. Mallikarjuna et al. [2] described that VAC works by exposing a wound to subatmospheric pressure for an extended period increasing blood supply, migration of inflammatory cells and the formation of granulation tissue compared with traditional dressing $[8,14]$.

Patients undergoing VAC reported longer durations of hospital stay in some studies [15-17], although some authors described opposite data [18, 19]. Yanaral et al. suggested that soft-tissue defects extension may impact the LOS which can be exacerbated by sepsis-induced complications or large soft-tissue defects extending [20]. Yücel et al. retrospectively analyzed $25 \mathrm{FG}$ patients underlining how the use of VAC is likely associated with the increased complexity of the lesion [15]. Hence, most of the available literature reports that VAC is effective in the management of large wounds with less pain, a lower patient discomfort and number of dressings and a greater mobility [13-21]. Furthermore, given the rarity of this clinical condition, all 


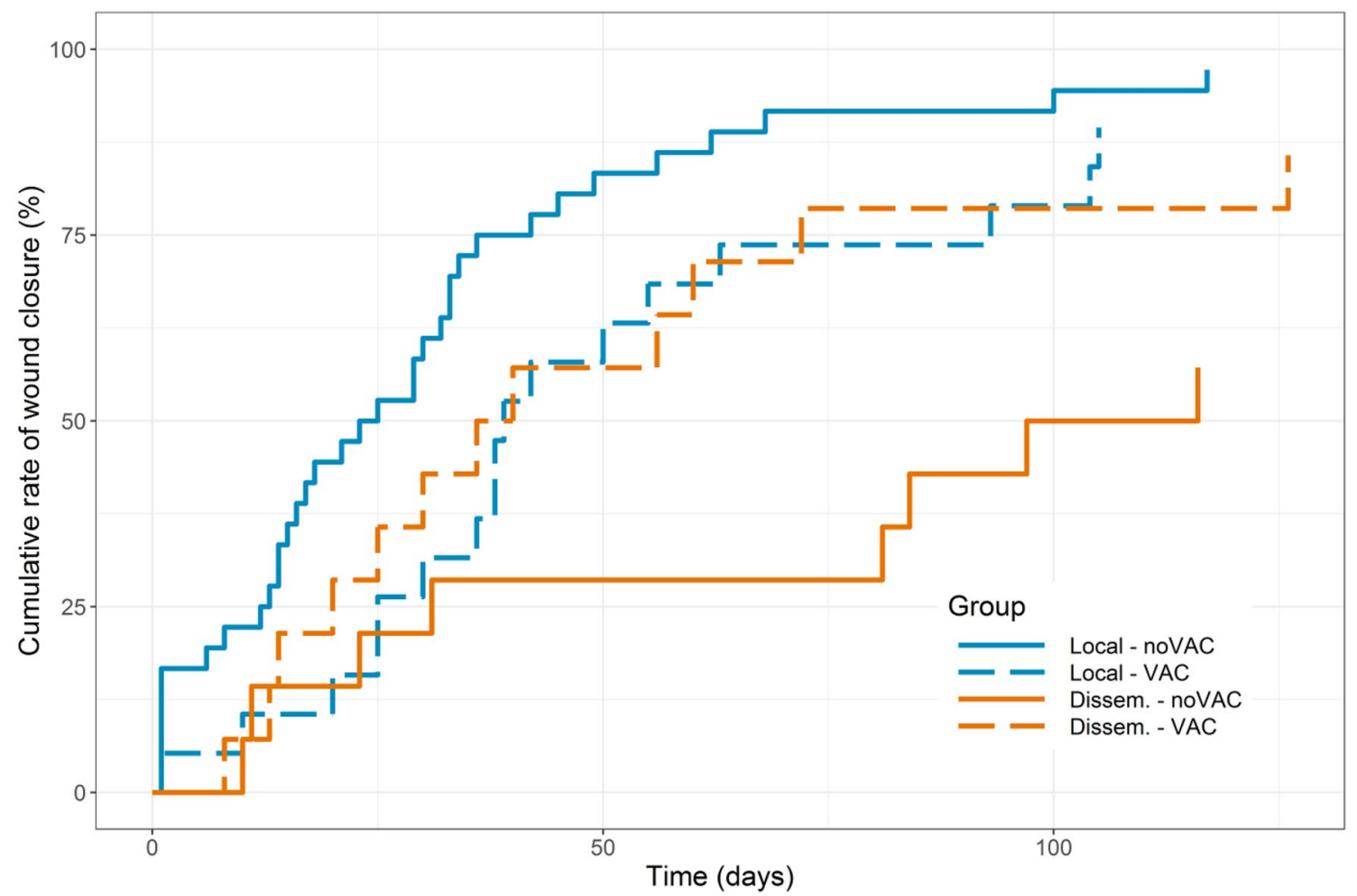

Fig. 2 Time trends of wound closure cumulative rates in patients with local and disseminated FG managed with VAC or no-VAC therapy

Fig. 3 Kaplan-Meier survival curves for the overall survival of patients with local and disseminated FG managed with VAC or no-VAC therapy

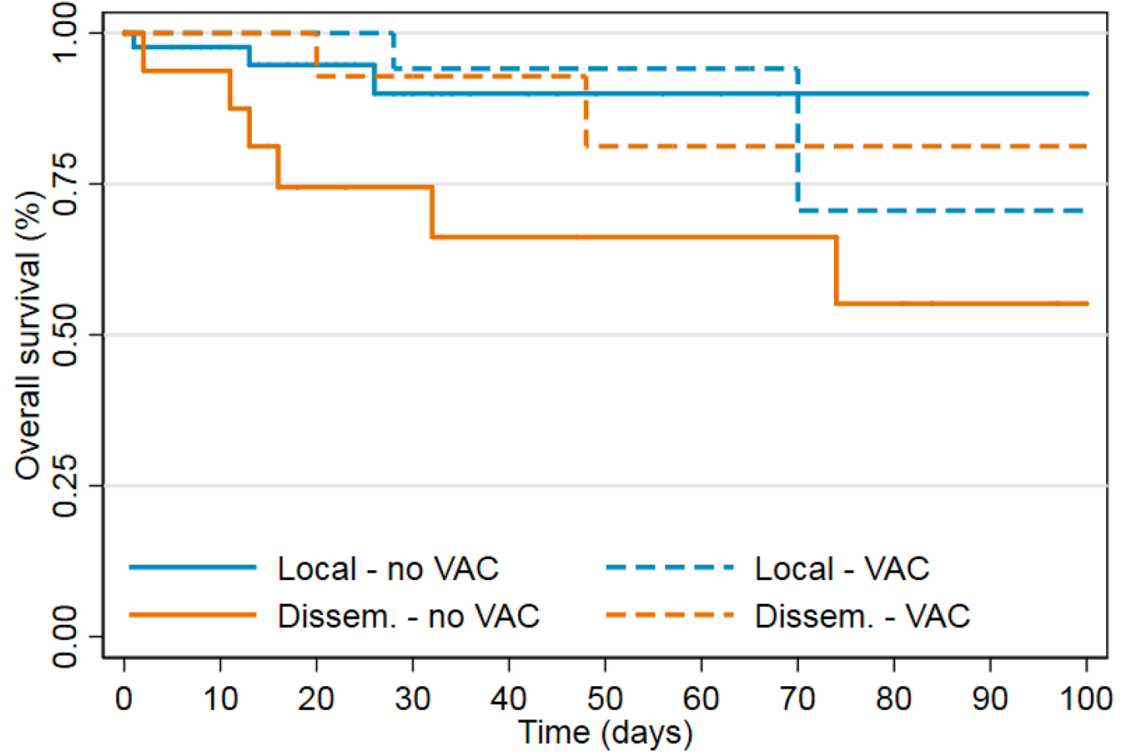

Num. at risk

Local - no VAC
Local - VAC
Dissem. - no VAC
Dissem. - VAC

\begin{tabular}{ll}
43 & 36 \\
19 & 18 \\
16 & 15 \\
\hline
\end{tabular}

25
17
10
14

$\begin{array}{cc}16 & 8 \\ 15 & 13 \\ 9 & 7 \\ 10 & 9\end{array}$

$\begin{array}{ll}5 & 4 \\ 8 & 7 \\ 6 & 6 \\ 7 & 6\end{array}$

$\begin{array}{ll}4 & 2 \\ 7 & 4 \\ 6 & 6 \\ 6 & 3\end{array}$

$\begin{array}{llll}2 & 2 & 2 & 2 \\ 4 & 3 & 3 & 3 \\ 6 & 5 & 3 & 2 \\ 3 & 3 & 3 & 3\end{array}$


the studies available about FG are retrospective and with small cohorts leading to low level evidence papers.

Alternative and/or complementary approach to VAC have been proposed. The role of hyperbaric oxygen (HBO) therapy remains controversial. Beneficial effects of HBO such as an increase in oxygen tension in the infected area have been reported [22-24] in very small case series ameliorating the tissue oxygenation [23]. However, HBO is an expensive treatment option without any described improvement in prognosis [25]. Therefore, Wagner et al. suggested an aggressive multimodality therapy based on surgery, antibiotics and VAC could be crucial where other approaches such as HBO may be more expensive and not so helpful [26]. VAC has been criticized to be expensive [27], Ozturk et al. performed a cost analysis reporting that the cost for treating five FG patients with VAC was $\$ 8,850$ versus $\$ 8,800$ for conventional treatment of five similar patients [11]. Although there were not significant differences in the costs, this was just a preliminary and not accurate estimation. Furthermore, health care systems are very different for each country and cost-analysis studies are mandatory.

Given these considerations, this multi-institutional observational study aimed to explore the possible role of VAC therapy versus conventional dressings in FG in terms of cumulative rate of wound closure and OS. Moreover, we analyzed a large cohort of FG cases collecting a wide spectrum of data concerning: predisposing factors, admission blood results, FG origin and extension, pathogens, urinary or fecal diversion, complications, LOS, type and number of surgical procedures, use of VAC or conventional dressing, time from initial debridement to wound closure. In our study, of 92 FG patients, 33 were treated with VAC (19 with local and 14 with disseminated disease) and 59 were treated with conventional approach (43 with local and 16 with disseminated disease). We considered secondary or tertiary (VACmediated) intention healings without reconstruction.

Within the nine centers, no-VAC therapy (otherwise called conventional $[13,14]$ ) had not a standardized protocol leading to the use of different antiseptic solutions in slightly but actually different manners. This limit should be stated given its alignment with the heterogeneity of the conventional therapy in the literature $[13,14]$. According to other papers [14-16], our results showed that VAC therapy is associated with a longer LOS both in local and disseminated FG.

Although the results on the time to complete wound closure did not show a significant difference between disseminated cases treated either with VAC or with the no-VAC approach, patients with a local disease reported a significantly longer recovery when managed with the VAC therapy. Nevertheless, in patients with disseminated disease treated with VAC we observed significant differences regarding the wound closure rate that may suggest an advantage in the use of this technique.
Ten weeks was chosen as a reference time for assessing wound closure arbitrarily given that two-thirds (61/92, $66.3 \%$ ) of the patients reported a tertiary wound closure at 10 weeks. Regarding the cumulative rate of wound closure, the results at 10 weeks after surgery did not show a statistically significant difference in the local FG group once treated with or without VAC. Conversely, the multivariable logistic regression analysis of 10 -weeks wound closure cumulative rate showed that the disseminated FG patients treated with VAC had a higher percentage of wound closure ( $p=0.036)$. Furthermore, comparing the KM Kaplan-Meier survival curves for the OS, a significant difference between patients with local and disseminated FG was found when patients were treated with conventional dressings (OS rate at 90 days $0.90,95 \%$ CI $0.71-0.97$ vs $0.55,95 \%$ CI $0.24-0.78$, respectively; $p=0.039$ ) and the Cox proportional hazard regression model highlighted that patients with disseminated FG managed with the no-VAC approach showed the lowest inter-group OS. Thus, our study suggests that VAC may offer an advantage in terms of wound closure at 10 weeks, especially for disseminated cases of FG where an aggressive multimodality approach can be crucial; these patients also have the lowest OS when not treated with VAC, suggesting its possible role in OS.

As we stated above, our purpose was to investigate the role of VAC in the multimodal approach for the FG in a hypothesis-generating framework. In other words, given the retrospective design of our study and the relatively small sample size, conclusions cannot be definitive on the role of VAC therapy on local or disseminated FG patients. Although this is not a prospective trial, the retrospective analysis brought out some elements that seemed to suggest an advantage in the VAC use in disseminated forms of disease in terms of faster recovery and a better OS. These data seem to suggest that in a patient presenting with a disseminated FG disease, VAC therapy may offer an advantage in the context of a multimodal approach. However, given the observational retrospective design of this study and its limited number of patients, we could not provide a decisionmaking algorithm. Anyhow, defining a clear algorithm was not our endpoint; this aim could be adequately addressed only by a hypothesis-testing randomized clinical trial powered with a proper sample size.

Although these results seemed to be encouraging, the limits of the study need to be carefully taken into account. We reviewed 92 patients and a wide set of variables with a hypothesis-generating and exploratory purpose. This retrospective design did not allow to perform a controlled treatment allocation, and this led to multiple and unavoidable biases (e.g., selection, information). The sample size is limited to 92 cases that are a relatively large sample for this rare pathology, but from a statistical point of view, they can represent a limit to the analysis. A prospective randomized 
well powered clinical study will help to better clarify the role of VAC in this niche of patients. Unfortunately, prospectively accrue and randomize patients is also difficult since this is typically an emergency situation and does not allow for detailed, thorough anamnesis and enrolling in a trial.

\section{Conclusions}

In patients with disseminated FG, VAC may offer an advantage in terms of 10-week wound closure cumulative rate and OS at 90 days after initial surgery. On the contrary, local FG patients did not show the same advantages.

These results in the use of VAC in FG need to be supported by a large randomized controlled trial.

Author contributions Protocol/project development: BP, CC, IV. Data analysis: SM, CC. Manuscript writing/editing: IV, CC, SM, BP. Data collection or management: BP, CC, IV, FR, FA, MS, RB, PS, FP, BG, MA, CA, PAL, SYA, AA, MT, VA, PF, CA, ZG, LC, PF, FAE.

\section{Compliance with ethical standards}

Conflict of interest The authors declare that they have no conflict of interest.

\section{References}

1. Fournier JA (1883) Gangrene foudroyante de la verge. Med Pract 4:589-597

2. Mallikarjuna MN, Vijayakumar A, Patil VS, Shivswamy BS (2012) Fournier's gangrene: current practices. ISRN Surg 2012:942437

3. Smith GL, Bunker CB, Dinneen MD (1998) Fournier's gangrene. Br J Urol 81(3):347-355

4. Sorensen MD, Krieger JN (2016) Fournier's gangrene: epidemiology and outcomes in the general US population. Urol Int 97:249

5. Stone HH, Martin JD Jr (1972) Synergistic necrotizing cellulitis. Ann Surg 175:702-711

6. Rodríguez Alonso A, Pérez García MD, Núñez López A, Ojea Calvo A, Alonso Rodrigo A, Rodríguez Iglesias B et al (2000) Fournier's gangrene: anatomo-clinical features in adults and children. Therapy update. Actas Urol Esp 24(4):294-306

7. Eke N (2000) Fournier's gangrene: a review of 1726 cases. Br J Surg 87:718-728

8. Chennamsetty A, Khourdaji I, Burks F, Killinger KA (2015) Contemporary diagnosis and management of Fournier's gangrene. Ther Adv Urol 7(4):203-215

9. Pastore AL, Palleschi G, Ripoli A, Silvestri L, Leto A, Autieri D et al (2013) A multistep approach to manage Fournier's gangrene in a patient with unknown type II diabetes: surgery, hyperbaric oxygen, and vacuum assisted closure therapy: a case report. J Med Case Rep 7:1
10. Bonkat G, Bartoletti RR, Bruyère F et al (2019) European Association of Urology - Infections in Urology Guidelines Panel, Copenhagen, EAU Guidelines Office, Arnhem. ISBN 978-94-92671-02-8

11. Ozturk E, Ozguc H, Yilmazlar T (2009) The use of vacuum assisted closure therapy in the management of Fournier's gangrene. Am J Surg 197(5):660-665 (discussion 665)

12. Hagedorn JC, Wessells H (2017) A contemporary update on Fournier's gangrene. Nat Rev Urol 14(4):205-214

13. Yanaral F, Balci C, Ozgor F, Simsek A, Onuk O, Aydin M et al (2017) Comparison of conventional dressings and vacuumassisted closure in the wound therapy of Fournier's gangrene. Arch Ital Urol Androl 89(3):208-211

14. Ozkan O, Koksal N, Altinli E, Celik A, Uzun M, Cikman O (2016) Fournier's gangrene current approaches. Int Wound J 13(5):713-716

15. Yücel M, Özpek A, Başak F, Kılıç A, Ünal E, Yüksekdağ S et al (2017) Fournier's gangrene: a retrospective analysis of 25 patients. Ulus Travma Acil Cerrahi Derg 23(5):400-404

16. Czymek R, Schmidt A, Eckmann C, Bouchard R, Wulff B, Laubert $\mathrm{T}$ et al (2009) Fournier's gangrene: vacuum-assisted closure versus conventional dressings. Am J Surg 197(2):168-176

17. Korkut M, Içöz G, Dayangaç M, Akgün E, Yeniay L, Erdoğan O et al (2003) Outcome analysis in patients with Fournier's gangrene: report of 45 cases. Dis Colon Rectum 46(5):649-652

18. Assenza M, Cozza V, Sacco E, Clementi I, Tarantino B, Passafiume F et al (2011) VAC (Vacuum Assisted Closure) treatment in Fournier's gangrene: personal experience and literature review. Clin Ter 162(1):e1-5

19. Cuccia G, Mucciardi G, Morgia G, Stagno d'Alcontres F, Galì A et al (2009) Vacuum-assisted closure for the treatment of Fournier's gangrene. Urol Int 82(4):426-431

20. Saijo S, Kuramoto Y, Yoshinari M (1990) Extremely extended Fournier's gangrene. Dermatologica 181:228-232

21. Oguz A, Gümüş M, Turkoglu A, Bozdăg Z, Ülger BV, Agaçayak E et al (2015) Fournier's gangrene: a summary of 10 years of clinical experience. Int Surg 100(5):934-941

22. Korhonen K (2000) Hyperbaric oxygen therapy in acute necrotizing infections with a special reference to the effects on tissue gas tensions. Ann Chir Gynaecol 89(suppl):7ff

23. Pizzorno R, Bonini F, Donelli A (1997) Hyperbaric oxygen therapy in the treatment of Fournier's disease in 11 male patients. J Urol 158:837-840

24. Capelli-Schellpfeffer M, Gerber GS (1999) The use of hyperbaric oxygen in urology. J Urol 162:647-654

25. Shupak A, Shoshani O, Goldenberg I (1995) Necrotizing fasciitis: an indication for hyperbaric oxygenation therapy? Surgery 118:873-878

26. Wagner S, Greco F, Hoda MR, Kawan F, Heynemann H, Fornara $P$ (2011) Is intensive multimodality therapy the best treatment for fournier gangrene? Evaluation of clinical outcome and survival rate of 41 patients. Surg Infect (Larchmt) 12(5):379-383

27. Lambert KV, Hayes P, McCarthy M (2005) Vacuum assisted closure: a review of development and current applications. Eur J Vasc Endovasc Surg 29:219-226

Publisher's Note Springer Nature remains neutral with regard to jurisdictional claims in published maps and institutional affiliations. 